\title{
Synthesis and Adsorption Performance of Molecularly Imprinted Microspheres for Quercetin by Suspension Polymerization
}

\author{
Yanbin Yun ${ }^{2}$, Minghang Zhu' ${ }^{1}$, Zhimiao Zhang ${ }^{1}$, Chao Liu ${ }^{1}$, Jiandu Lei ${ }^{1 *}$, Guanghui Ma ${ }^{1}$ and Zhiguo Su${ }^{1}$
}

${ }^{1}$ State Key Laboratory of Biochemical Engineering, Institute of Process Engineering, Chinese Academy of Sciences, Beijing 100190, P.R.China

${ }^{2}$ College of Environmental Science and Engineering, Beijing Forestry University, Beijing 100083, P.R.China

\begin{abstract}
The preparation of molecularly imprinted microspheres for quercetin by aqueous suspension polymerization was first presented, in which quercetin is used as template molecule, methacrylic acid as functional monomer and ethylene glycol dimethacrylate as cross-linker. Morphological of the imprinted microspheres for quercetin was characterized by scanning electron microscopy. The imprinting effect of the imprinted microspheres was evaluated, and selectivity analysis suggests that the imprinted microspheres can selectively recognize quercetin from its structure analogues. In addition, adsorption kinetics and adsorption isotherm are used to investigate the binding characteristics of the imprinted microspheres. Results indicate that quercetin can be adsorbed rapidly by the imprinted microspheres, and the maximum theoretical static binding capacity is up to $96.5927 \mathrm{mg} \mathrm{g}^{-1}$.
\end{abstract}

Keywords: Molecular imprinting; Microspheres; Quercetin; Recognition

\section{Introduction}

Quercetin (3,3',4,5,7-penta-hydroxy flavone) is a plant-derived flavonoid found widely occurring in leaves, fruits, vegetables and grains [1]. Quercetin has become a hot topic based on its various bioactivities such as anti-inflammation, inoxidability, antiviral, antitumor and innate immune function [2]. The analysis of quercetin involve in HPLC-UV [3-5], electrogenerated chemiluminescence [6] and capillary electrophoresis [7]. Because of low concentrations of quercetins in nature, the complexity of samples and the structural similarity to other flavonols, selective extract methods are necessary prior to analysis.

Molecularly imprinted polymers (MIPs) possess high selectivity toward the target molecules (or the template molecules) due to the "lock-key" relationship between MIPs and the target molecules [8-10]. MIPs have been widely applied in drug separation, food and environmental testing, antibody or receptor analogues, sensors and many other fields, which have shown good prospects [11-19].

Some studies about determination and separation for quercetin using MIPs have been reported [4,20-22]. However, these quercetinMIPs were prepared by bulk polymerization, and the required particles were obtained with crushing, grinding and sieving. As well as we known, the bulk polymerization method has many limitations, such as time-consuming, waste, homogeneous absorption sites, poor reproducibility and selectivity [23].

In this work, the preparation of molecularly imprinted microspheres for quercetin by aqueous suspension polymerization was first presented. The imprinting effect of the imprinted microspheres was evaluated, and selectively recognition capability was demonstrated. The equilibrium binding experiment shows that the maximum theoretical static binding capacity of the imprinted microspheres for quercetin can be up to $96.5927 \mathrm{mg} \mathrm{g}^{-1}$.

\section{Materials and Methods}

\section{Reagents}

Quercetin(QCT), isorhamnetin(IRT), rutin(RUT) and ethylene glycol dimethacrylate (EGDMA) were obtained from Sigma-Aldrich (USA, AR). Methacrylic acid (MAA) was provided by West Long Chemical Plant (China, AR). Polyvinyl alcohol (PVA) was obtained from Kuraray Chemical Co., Ltd (Japan, DP 1700). Ethyl acetate, acetic acid, and methanol were from Beijing Chemical Plant (China, AR). The free radical initiator, 2, 2-azobis (2-isobutyronitrile) (AIBN), was supplied by Shanghai Reagent four plants (China, AR).

Preparation of molecularly imprinted microspheres for quercetin

Molecularly imprinted microspheres for quercetin were prepared by aqueous suspension polymerization methods as follows: quercetin, MAA, EGDMA and AIBN were dissolved in porogen solvent ethyl acetate (also used as oil phase). Then it was purged with high purity nitrogen for $20 \mathrm{~min}$, and added into $1.5 \% \mathrm{wt}$. PVA/water in a glass vessel and stirred at $350 \mathrm{rpm}$. Polymerization was carried out at $60^{\circ} \mathrm{C}$ for $24 \mathrm{~h}$ under stirring and $\mathrm{N}_{2}$ atmosphere.

The resulted microspheres were washed by hot water to remove PVA. Then they were washed respectively by $15 \%$ acetic acid-methanol and methanol/water solution under ultrasonic until no quercetin was detected in the rinse solution.

Non-imprinted polymers (NIPs) for control experiments were obtained following the procedure described above but excluding quercetin from the formulation.

\section{Characterization of microspheres}

For scanning electron microscope (SEM) observation, the obtained

*Corresponding author: Jiandu Lei, Institute of Process Engineering, Chinese Academy of Sciences. No.1, 2st North Street, Zhongguancun, Haidian District, Beijing 100190, P.R.China, E-mail: jdlei@home.ipe.ac.cn

Received October 10, 2011; Accepted March 22, 2012; Published March 24, 2012

Citation: Yun Y, Zhu M, Zhang Z, Liu C, Lei J, et al. (2012) Synthesis and Adsorption Performance of Molecularly Imprinted Microspheres for Quercetin by Suspension Polymerization. Pharm Anal Acta 3:151. doi:10.4172/2153-2435.1000151

Copyright: (C) 2012 Yun Y, et al. This is an open-access article distributed under the terms of the Creative Commons Attribution License, which permits unrestricted use, distribution, and reproduction in any medium, provided the original author and source are credited. 
Citation: Yun Y, Zhu M, Zhang Z, Liu C, Lei J, et al. (2012) Synthesis and Adsorption Performance of Molecularly Imprinted Microspheres for Quercetin by Suspension Polymerization. Pharm Anal Acta 3:151. doi:10.4172/2153-2435.1000151

microspheres were dried at $60^{\circ} \mathrm{C}$ and then attached to the silver paper and coated with a gold layer. A scanning electron microscope (JSM6700, JEOL SEM Co., Japan) was used for the morphology observation.

\section{HPLC-UV analysis of analytes}

The HPLC-UV analysis was carried out using a Shimadzu-LC20AT pump equipped with SIL-20A injector, a Shimadzu SPD-M20A US detector and a CTO-2AScolumn oven. Column oven temperature is controlled at $25^{\circ} \mathrm{C}$. The column is $\mathrm{C} 18$ column $(4.6 \mathrm{~mm} \times 250 \mathrm{~mm}$, i.d. $)$. Analytical procedure was conducted according to the reference [22]. That is, the column was equilibrated by mobile phase that acetonitrile and $0.1 \%(\mathrm{w} / \mathrm{v}) \mathrm{H}_{3} \mathrm{PO}_{4}$ aqueous solution $(36: 64, \mathrm{v} / \mathrm{v})$. The detection was run with wavelength at $365 \mathrm{~nm}$. The temperature was controlled at $25^{\circ} \mathrm{C}$. The flow rate was maintained at $0.6 \mathrm{~mL} \mathrm{~min}^{-1}$ and $20 \mu \mathrm{L}$ of sample was injected.

\section{Binding analysis of molecularly imprinted microspheres}

The imprinted microspheres or non-imprinted microspheres of $25 \mathrm{mg}$ were mixed with $5 \mathrm{~mL}$ of solution containing a known concentration of quercetin in methanol/water $(95: 5, \mathrm{v} / \mathrm{v})$. The mixture was stirred at $150 \mathrm{rpm}$ and incubated for $3 \mathrm{~h}$ at room temperature. The concentration of the free substrate in the supernatant solution was determined by HPLC-UV analysis after the sample was centrifuged at $6000 \mathrm{rpm}$ for $12 \mathrm{~min}$. The binding capacity (Q) was defined as $\mathrm{mg}$ of substrate bound per $1 \mathrm{~g}$ microspheres, and calculated by the change of quercetin concentration after and before adsorption by equation (1), in which $C_{0}\left(\mathrm{mg} \mathrm{mL}^{-1}\right)$ and $C\left(\mathrm{mg} \mathrm{mL}^{-1}\right)$ are the initial concentration and free concentration of substrate in the supernatant, respectively. $V(\mathrm{~mL})$ is the volume of adsorption solution. $W(\mathrm{~g})$ is the mass of the microspheres.

$$
Q=\left(C_{0}-C\right) \times V / W
$$

\section{Selectivity of the imprinted microspheres}

Three kinds of flavones (quercetin, isorhamnetin, rutin) were selected as competitive agents to estimate selectivity of MIPs for quercetin. Structures of quercetin, isorhamnetin and rutin were shown in Figure 1. MIP/NIP microspheres $(25 \mathrm{mg}$ ) were respectively dispersed in 6 vials containing $5 \mathrm{~mL}$ of methanol/water $(95: 5$, v/v) solution of the substrate. And initial concentrations of quercetin, isorhamnetin and rutin are $80 \mathrm{mg} \mathrm{L}^{-1}$. The mixture is stirred at $150 \mathrm{rpm}$ and incubated for $3 \mathrm{~h}$ at room temperature, and equilibrium concentrations of quercetin, isorhamnetin and rutin are determined by HPLC-UV analysis.

\section{Results and Discussion}

\section{Preparation and evaluation of molecularly imprinted microspheres}

There are a few reports about the preparation of MIPs for quercetin, but these MIPs were prepared by bulk polymerization followed by grinding to particles. These imprinted particles are polydisperse, irregular and containing a large portion of fine particles. Furthermore, extensive sieving and sediment are necessary in order to achieve a rather narrower size distribution and remove fine particles. They are time-consuming and labor-wasting, and the imprinting effect is always not satisfactory [23].

In this work, the imprinted microspheres for quercetin were prepared by aqueous suspension polymerization, in which water solution containing 1.5\% PVA was used as continuous phase, and ethyl acetate solution (it contains quercetin, MAA, EGDMA and AIBN) was

used as disperse phase. The ratio of the template molecule quercetin to functional monomer MAA was optimized. The composition of prepolymerization mixtures and imprinting factor (IF) for MIPs and NIPs are shown in Table 1.

These results indicate that when the ratio of quercetin to MAA is $1: 4$, the imprinting effect is the best, and the imprinting factor (the amount of quercetin bound by MIPs / the amount of quercetin bound by NIPs) is the highest. It can be explained as follows: when the ratio of quercetin to MAA during 1:1 1:3, IF is lower because the insufficient amount of functional monomers in MIPs leads to less binding sites. On the other hand, while the ratio of quercetin to MAA is 1:5 and 1:6, IF is also lower because that the excessive functional monomer MAA in MIPS make non-selective binding sites increase, which creates the enhancement of non-specific interaction between quercetin and MAA. So, the ratio of template quercetin to functional monomer MAA is 1:4 in the following experiments.

\section{Characterization of microspheres}

The imprinted microspheres were detected by scanning electron microscope (SEM), and the results were shown in Figure 2. SEM micrographs of the imprinted microspheres reveal that the imprinted microspheres have good sphericity and smooth surface, and there are many micropores in the surface of the microspheres.<smiles>O=c1c(O)c(-c2ccc(O)c(O)c2)oc2cc(O)cc(O)c12</smiles>

Quercetin

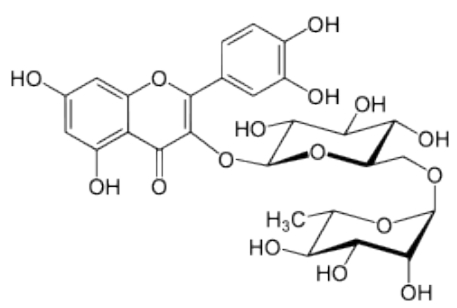

Rutin

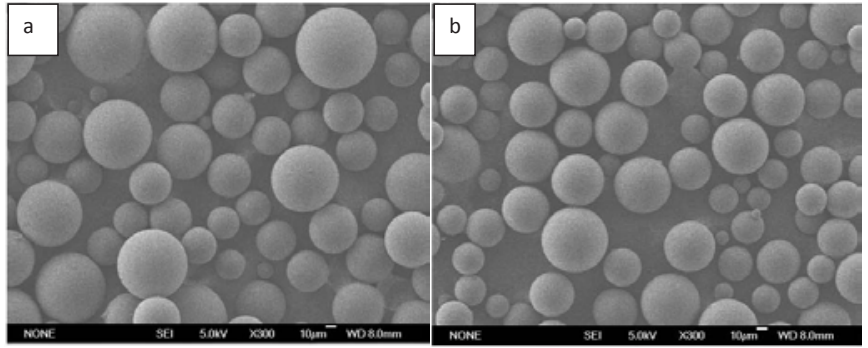

Figure 2: Morphological characterization of the microspheres. (a) Scanning electron micrographs of MIPs; (b) Scanning electron micrographs of NIP. 
Citation: Yun Y, Zhu M, Zhang Z, Liu C, Lei J, et al. (2012) Synthesis and Adsorption Performance of Molecularly Imprinted Microspheres for Quercetin by Suspension Polymerization. Pharm Anal Acta 3:151. doi:10.4172/2153-2435.1000151

\section{Selectivity of the imprinted microspheres}

In order to study the recognition capability of the imprinted microspheres, the binding amounts for quercetin, isorhamnetin and rutin by the imprinted microspheres were determined using HPLCUV analysis. The results are shown in Figure 3, and it indicates that the binding amounts of MIPs for quercetin is much more than that of isorhamnetin and rutin, which further demonstrates the imprinted microspheres have good selective recognition for the template molecule quercetin. The reason is that strong hydrogen bonds are formed between $-\mathrm{OH}$ of quercetin and $-\mathrm{COOH}$ of MAA in the solvent. In addition, Schematic diagram of the interaction between quercetin and MAA is shown in Figure 4.

\section{Adsorption kinetics of the imprinted microspheres for quercetin}

The adsorption kinetics behavior of the imprinted microspheres for quercetin was investigated by changing the adsorption time from $0 \mathrm{~min}$ to $180 \mathrm{~min}$. The initial concentration of quercetin was kept a constant of $80 \mathrm{mg} \mathrm{L}^{-1}$ in methanol/water (95: 5, v/v). Adding $25 \mathrm{mg}$ of microspheres into $5 \mathrm{~mL}$ solution, then they were mixed at room temperature under stirring at $150 \mathrm{rpm}$. After being absorbed for some time, each sample was centrifuged and $20 \mu \mathrm{L}$ of supernatant was assessed by HPLC-UV analysis.

The adsorption dynamic curves of MIPs and NIPs were shown in Figure 5. It can be seen that the amount of quercetin binding to the MIPs is much higher than that of the NIPs. Moreover, to the MIPs, there exists a rapid dynamic adsorption of quercetin to the MIPs. In the first $40 \mathrm{~min}$, the adsorbed amount increases fast with the increase of adsorption time, and then the adsorbed amount keeps a constant in the succedent time. However, there is no large difference among the binding amount of the NIPs for quercetin with the time.

\section{Adsorption isotherm of the imprinted microspheres}

It is very important to investigate adsorption isotherm of the imprinted microspheres for further study. The method usually utilized to investigate the thermodynamic adsorption properties of MIPs is to plot an adsorption curve. The experiments were carried out in various quercetin concentrations $\left(1.0 \times 10^{-4} \sim 50 \times 10^{-4} \mathrm{~mol} \mathrm{~L}^{-1}\right)$ in methanol/ water (95: 5, v/v), in which $25 \mathrm{mg}$ of microspheres (MIP4 and NIP4 in the Table 1) were mixed with $5 \mathrm{~mL}$ of quercetin solution for $3 \mathrm{~h}$.

Figure 6a shows adsorption isotherms of MIPs and NIPs. The results suggest the binding amounts of MIPs and NIPs for quercetin improve with the increase of concentration of quercetin. Moreover, the former is always much larger than the latter. When initial concentration of quercetin is $30 \times 10^{-4} \mathrm{~mol} \mathrm{~L}^{-1}$, the adsorbed amount is about $77 \mathrm{mg} \mathrm{g}^{-1}$ toward the MIPs and $23 \mathrm{mg} \mathrm{g}^{-1}$ toward the NIPs, respectively, and the imprinting factor is 3.47 , it indicates that the obtained MIPs by our

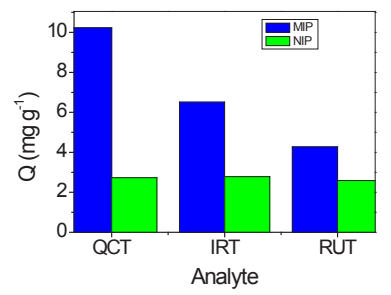

Figure 3: Selectivity of the imprinted microspheres.<smiles>C=C(C)C(=O)OC(C)C(=O)O</smiles>

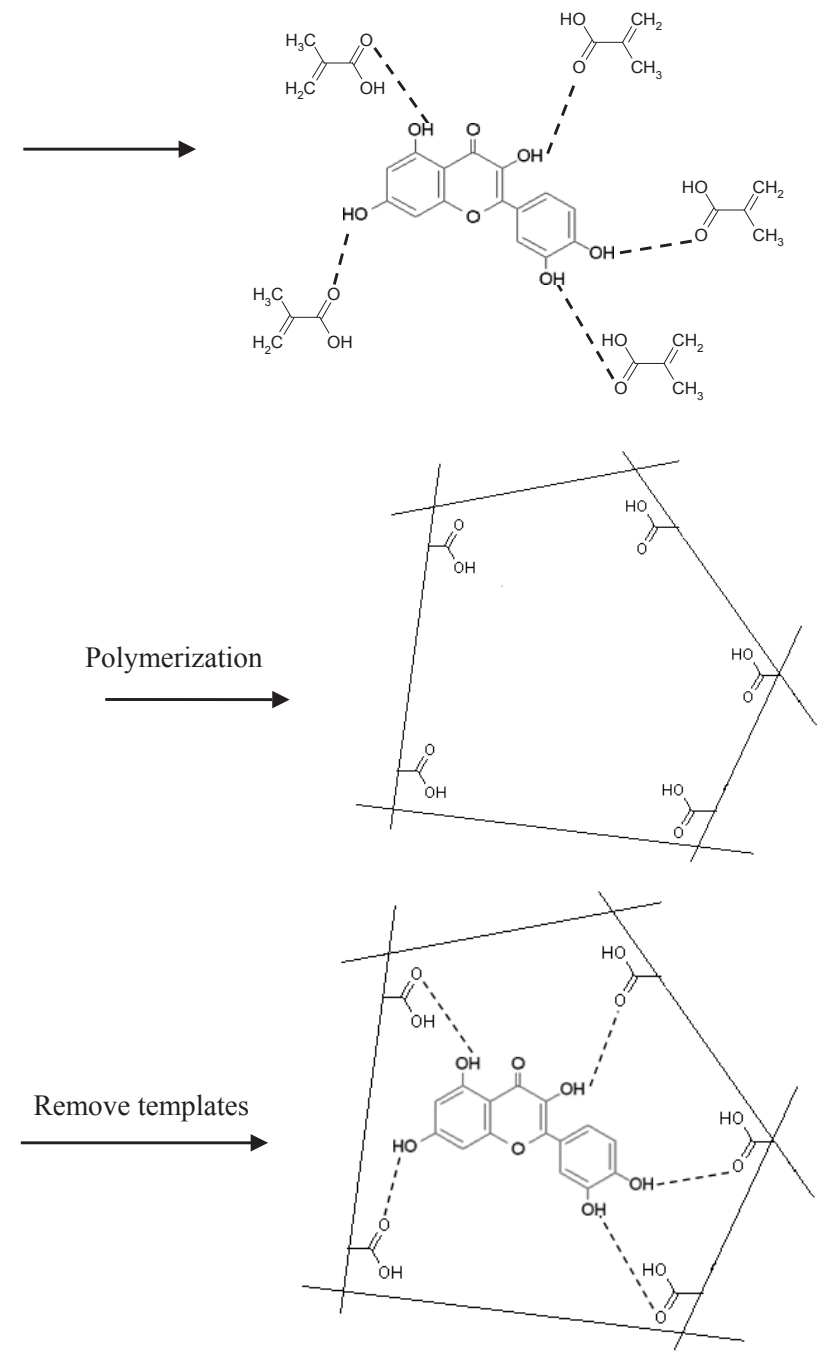

Figure 4: Schematic diagram of the interaction between quercetin and MAA.

methods have high specific adsorption for the template molecule. It is because that there are apparent differences in tri-dimensional structure between the MIPs and the NIPs. In the MIPs, there are a lot of sites and cavities which are complementary to the template quercetin in size and shape, and they are contributive to high effective selectivity for the template. To NIPs, however, there are no sites and cavities complementary to the template and so their selectivity for quercetin is worse.

In addition, the plotted adsorption isotherm curve of MIPs is shown in Figure 6b. The plotted curve was approximated by modified Langmuir isotherm (equation 2) with parameters $a=96.5927, b=0.0173$ and $c=-0.6340$, and the correlative coefficient $R^{2}=0.9951$. The result 
Citation: Yun Y, Zhu M, Zhang Z, Liu C, Lei J, et al. (2012) Synthesis and Adsorption Performance of Molecularly Imprinted Microspheres for Quercetin by Suspension Polymerization. Pharm Anal Acta 3:151. doi:10.4172/2153-2435.1000151

Page 4 of 5

indicates that the theoretical maximum absorption capacity of MIPs is up to $96.5927 \mathrm{mg} \mathrm{g}^{-1}$.

$$
y=\frac{a b x^{1-c}}{1+b x^{1-c}}
$$

\section{Conclusion}

The molecularly imprinted microspheres for quercetin was prepared by using suspension polymerization, in which $1.5 \%$ PVA-water solution is used as continuous phase, ethyl acetate solution including quercetin, MAA, EGDMA and AIBN used as disperse phase. The optimal molar ratio of quercetin to MAA is $1: 4$. The imprinted microspheres were characterized by scanning electron microscopy. Furthermore, the imprinting effect and selectivity of the imprinted microspheres were

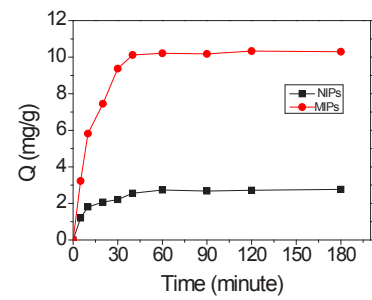

Figure 5: Adsorption dynamic curve of the MIPs and NIPs.

\begin{tabular}{|c|c|c|c|c|c|c|}
\hline No. & Polymers & Ratio $^{a}$ & QCT (mmol) & MAA(mmol) & $\mathrm{Q}\left(\mathrm{mg} \mathrm{g}^{-1}\right)$ & IF \\
\hline & MIP1 & $1: 1: 20$ & 0.5 & 0.5 & 8.12 & \multirow{2}{*}{2.59} \\
\hline & NIP1 & $1: 1: 20$ & 0.5 & 0.5 & 3.13 & \\
\hline & MIP2 & $1: 2: 20$ & 0.5 & 1.0 & 8.67 & \multirow{2}{*}{2.95} \\
\hline & NIP2 & $1: 2: 20$ & 0.5 & 1.0 & 2.93 & \\
\hline & MIP3 & $1: 3: 20$ & 0.5 & 1.5 & 9.15 & \multirow{2}{*}{3.22} \\
\hline & NIP3 & $1: 3: 20$ & 0.5 & 1.5 & 2.84 & \\
\hline & MIP4 & $1: 4: 20$ & 0.5 & 2.0 & 10.17 & \multirow{2}{*}{3.68} \\
\hline & NIP4 & $1: 4: 20$ & 0.5 & 2.0 & 2.76 & \\
\hline & MIP5 & $1: 5: 20$ & 0.5 & 2.5 & 9.48 & \multirow{2}{*}{3.41} \\
\hline & NIP5 & $1: 5: 20$ & 0.5 & 2.5 & 2.78 & \\
\hline & MIP6 & $1: 6: 20$ & 0.5 & 3.0 & 8.39 & \multirow{2}{*}{2.98} \\
\hline & NIP6 & $1: 6: 20$ & 0.5 & 3.0 & 2.81 & \\
\hline
\end{tabular}

The molar ratio of QCT to MAA to EGDMA.

Table 1: Composition of prepolymerization mixtures and IF for MIPs and NIPs.
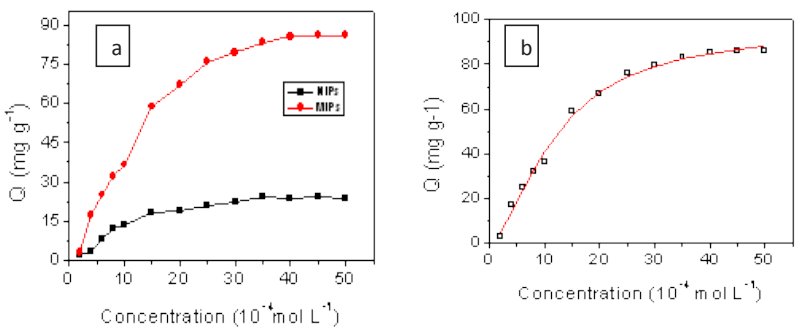

Figure 6: Absorption isotherm curve of MIPs and NIPs (a) The static equilibrium adsorption isotherm of MIPs and NIPs microspheres; (b) The plotted curve obtained using the data of adsorption capacity of MIPs. investigated, and the results show that the imprinted microspheres can specifically recognize quercetin from other three kinds of antibiotics. The equilibrium binding experiments were employed to study the binding behavior of the imprinted microspheres. The results indicate that the maximum theoretical static binding capacity of molecularly imprinted microspheres can be up to $96.5927 \mathrm{mg} \mathrm{g}^{-1}$.

Therefore, the resulted imprinted microspheres for quercetin have good selectivity and high binding capacity, and they are promising to be used as adsorption media, even also used as chromatographic stationary phase.

\section{Acknowledgement}

The authors are indebted to the State Forestry Administration 948 Innovative Significant Project (No. 2009-4-62), the National Natural Science Foundation of China (No. 20976179) and Beijing Natural Science Foundation (No. 2092027) for supporting this research

\section{References}

1. Wang YF, Wang XH, Zhu YT (2003) Advancement of researches in quercetin. Nat Prod Res Dev 15: 171-173

2. Russo N, Toscano M, Uccella N (2000) Semiempirical molecular modeling into quercetin reactive site: Structural, conformational, and electronic features. J Agric Food Chem 48: 3232-3237.

3. Zhu L, Xu X (2003) Selective separation of active inhibitors of epidermal growth factor receptor from Caragana Jubata by molecularly imprinted solid-phase extraction. J Chromatogr A 991: 151-158.

4. Xie J, Zhu L, Luo H, Zhou L, Li C, et al. (2001) Direct extraction of specific pharmacophoric flavonoids from ginko leaves using a molecularly imprinted polymer for quercetin. J Chromatogr A 934: 1-11.

5. Theodoridis G, Lasakova M, Skerikova V, Tegou A, Giantsiou N, et al. (2006) Molecular imprinting of natural flavonoid antioxidants: application in solidphase extraction for the sample pretreatment of natural products prior to HPLC analysis. J Sep Sci 29: 2310-2321.

6. Lei R, Xu X, Yu F, Li N, Liu HW, et al. (2008) A method to determine quercetin by enhanced luminol electrogenerated chemiluminescence (ECL) and quercetin autoxidation. Talanta 75: 1068-1074.

7. Zhang S, Dong S, Chi L, He P, Wang Q, et al. (2008) Simultaneous determination of flavonoids in chrysanthemum by capillary zone electrophoresis with running buffer modifiers. Talanta 76: 780-784.

8. Wulff G, Sarhan A, Zabrocki K (1973) Enzyme-analogue built polymers and their use for the resolution of racemates. Tetrahedron Lett 14: 4329-4332.

9. Norrlow O, Glad M, Mosbach K (1984) Acrylic polymer preparations containing recognition sites obtained by imprinting with substrates. J Chromatogr A 299 29-41.

10. Yoshimatsu K, Reimhult K, Krozer A, Mosbach K, Sode K, et al. (2007) Uniform molecularly imprinted microspheres and nanoparticles prepared by precipitation polymerization: the control of particle size suitable for different analytical applications. Anal Chim Acta 584: 112-121.

11. Lei JD, Tan TW (2002) Enantioselective separation of naproxen and investigation of affinity chromatography model using molecular imprinting. Biochem Eng J 11: 175-179.

12. Tamayo FG, Turiel E, Martin-Esteban A (2007) Molecularly imprinted polymers for solid-phase extraction and solid-phase microextraction: recent developments and future trends. J Chromatogr A 1152: 32-40.

13. Hung CY, Huang YT, Huang HH, Hwang CC (2006) HPLC separation of ibuprofen and mefenamic acid using molecularly imprinted polymer as stationary phase. E-Polymers 065: 1-10.

14. Curcio M, Puoci F, Cirillo G, lemma F, Spizzirri UG, et al. (2010) Selective Determination of Melamine in Aqueous Medium by Molecularly Imprinted Solid Phase Extraction. J Agric Food Chem 58: 11883-11887.

15. Luo W, Zhu L, Yu C, Tang H, Yu H, et al. (2008) Synthesis of surface molecularly 
Citation: Yun Y, Zhu M, Zhang Z, Liu C, Lei J, et al. (2012) Synthesis and Adsorption Performance of Molecularly Imprinted Microspheres for Quercetin by Suspension Polymerization. Pharm Anal Acta 3:151. doi:10.4172/2153-2435.1000151

Page 5 of 5

imprinted silica micro-particles in aqueous solution and the usage for selective off-line solid-phase extraction of 2, 4-dinitrophenol from water matrixes. Anal Chim Acta 618: 147-156

16. Lu Y, Li C, Zhang H, Liu X (2003) Study on the mechanism of chiral recognition with molecularly imprinted polymers. Anal Chim Acta 489: 33- 43.

17. Avila M, Zougagh M, Escarpa A, Rios A (2008) Molecularly imprinted polymers for selective piezoelectric sensing of small molecules. Trends Analyt Chem 27: 54-65.

18. Lavignac N, Allender CJ, Brain KR (2004) Current status of molecularly imprinted polymers as alternatives to antibodies in sorbent assays. Anal Chim Acta 510: 139-145.

19. Sergeyeva TA, Slinchenko OA, Gorbach LA, Matyushov VF, Brovko OO, et al. (2010) Catalytic molecularly imprinted polymer membranes: development of the biomimetic sensor for phenols detection. Anal Chim Acta 659: 274-279.
20. Yan LS, Jing J, Huang ZM, Wen ZD, Liu FT (2006) Preparation of quercetinimprinted polymer and its property as a sorbent for solid phase extraction. Chin J Anal Lab 25: 97-100.

21. Song X, Li J, Wang J, Chen L (2009) Quercetin molecularly imprinted polymers: Preparation, recognition characteristics and properties as sorbent for solidphase extraction. Talanta 80: 694-702.

22. O'Mahony J, Wei S, Molinelli A, Mizaikoff B (2006) Imprinted polymeric materials. Insight into the nature of prepolymerization complexes of quercetin imprinted polymers. Anal Chem 78: 6187-6190.

23. Wang J, Cormack PAG, Sherrington DC, Khoshdel E (2007) Synthesis and characterization of micrometer-sized molecularly imprinted spherical polymer particulates prepared via precipitation polymerization. Pure Appl Chem 79 1505-1519. 